BABAR-CONF-04/008

SLAC-PUB-10660

hep-ex/0408094

August, 2004

\title{
Measurement of the Branching Ratio $\Gamma\left(D_{s}^{*+} \rightarrow D_{s}^{+} \pi^{0}\right) / \Gamma\left(D_{s}^{*+} \rightarrow D_{s}^{+} \gamma\right)$
}

The BABAR Collaboration

August 18, 2004

\begin{abstract}
The $(\Delta I=1)$ decay $D_{s}^{*+} \rightarrow D_{s}^{+} \pi^{0}$ and $(\Delta I=0)$ decay $D_{s}^{*+} \rightarrow D_{s}^{+} \gamma$ have been reconstructed using $90.4 \mathrm{fb}^{-1}$ of data recorded by the BABAR detector at the PEP-II asymmetric-energy $e^{+} e^{-}$ collider. The relative branching fraction of isospin violating decay to isospin conserving decay has been measured. Our preliminary result for this ratio is $\Gamma\left(D_{s}^{*+} \rightarrow D_{s}^{+} \pi^{0}\right) / \Gamma\left(D_{s}^{*+} \rightarrow D_{s}^{+} \gamma\right)=$ $0.0621 \pm 0.0049$ (stat) \pm 0.0063 (syst). In addition we reconstruct the decays $D^{* 0} \rightarrow D^{0} \pi^{0}$ and $D^{* 0} \rightarrow D^{0} \gamma$ for completeness and measure a preliminary relative branching fraction to be $\Gamma\left(D^{* 0} \rightarrow\right.$ $\left.D^{0} \pi^{0}\right) / \Gamma\left(D^{* 0} \rightarrow D^{0} \gamma\right)=1.740 \pm 0.020$ (stat) \pm 0.125 (syst). Both measurements represent significant improvements over present world averages.
\end{abstract}

Submitted to the $32^{\text {nd }}$ International Conference on High-Energy Physics, ICHEP 04, 16 August-22 August 2004, Beijing, China

Stanford Linear Accelerator Center, Stanford University, Stanford, CA 94309

Work supported in part by Department of Energy contract DE-AC03-76SF00515. 
The BABAR Collaboration,

B. Aubert, R. Barate, D. Boutigny, F. Couderc, J.-M. Gaillard, A. Hicheur, Y. Karyotakis, J. P. Lees, V. Tisserand, A. Zghiche

Laboratoire de Physique des Particules, F-74941 Annecy-le-Vieux, France

A. Palano, A. Pompili

Università di Bari, Dipartimento di Fisica and INFN, I-70126 Bari, Italy

J. C. Chen, N. D. Qi, G. Rong, P. Wang, Y. S. Zhu

Institute of High Energy Physics, Beijing 100039, China

G. Eigen, I. Ofte, B. Stugu

University of Bergen, Inst. of Physics, N-5007 Bergen, Norway

G. S. Abrams, A. W. Borgland, A. B. Breon, D. N. Brown, J. Button-Shafer, R. N. Cahn, E. Charles, C. T. Day, M. S. Gill, A. V. Gritsan, Y. Groysman, R. G. Jacobsen, R. W. Kadel, J. Kadyk, L. T. Kerth, Yu. G. Kolomensky, G. Kukartsev, G. Lynch, L. M. Mir, P. J. Oddone, T. J. Orimoto, M. Pripstein, N. A. Roe, M. T. Ronan, V. G. Shelkov, W. A. Wenzel

Lawrence Berkeley National Laboratory and University of California, Berkeley, CA 94720, USA

M. Barrett, K. E. Ford, T. J. Harrison, A. J. Hart, C. M. Hawkes, S. E. Morgan, A. T. Watson University of Birmingham, Birmingham, B15 2TT, United Kingdom

M. Fritsch, K. Goetzen, T. Held, H. Koch, B. Lewandowski, M. Pelizaeus, M. Steinke Ruhr Universität Bochum, Institut für Experimentalphysik 1, D-44780 Bochum, Germany

J. T. Boyd, N. Chevalier, W. N. Cottingham, M. P. Kelly, T. E. Latham, F. F. Wilson University of Bristol, Bristol BS8 1TL, United Kingdom

T. Cuhadar-Donszelmann, C. Hearty, N. S. Knecht, T. S. Mattison, J. A. McKenna, D. Thiessen University of British Columbia, Vancouver, BC, Canada V6T $1 Z 1$

A. Khan, P. Kyberd, L. Teodorescu Brunel University, Uxbridge, Middlesex UB8 3PH, United Kingdom

A. E. Blinov, V. E. Blinov, V. P. Druzhinin, V. B. Golubev, V. N. Ivanchenko, E. A. Kravchenko, A. P. Onuchin, S. I. Serednyakov, Yu. I. Skovpen, E. P. Solodov, A. N. Yushkov

Budker Institute of Nuclear Physics, Novosibirsk 630090, Russia

D. Best, M. Bruinsma, M. Chao, I. Eschrich, D. Kirkby, A. J. Lankford, M. Mandelkern, R. K. Mommsen, W. Roethel, D. P. Stoker University of California at Irvine, Irvine, CA 92697, USA

C. Buchanan, B. L. Hartfiel

University of California at Los Angeles, Los Angeles, CA 90024, USA

S. D. Foulkes, J. W. Gary, B. C. Shen, K. Wang

University of California at Riverside, Riverside, CA 92521, USA 
D. del Re, H. K. Hadavand, E. J. Hill, D. B. MacFarlane, H. P. Paar, Sh. Rahatlou, V. Sharma University of California at San Diego, La Jolla, CA 92093, USA

J. W. Berryhill, C. Campagnari, B. Dahmes, O. Long, A. Lu, M. A. Mazur, J. D. Richman, W. Verkerke University of California at Santa Barbara, Santa Barbara, CA 93106, USA

T. W. Beck, A. M. Eisner, C. A. Heusch, J. Kroseberg, W. S. Lockman, G. Nesom, T. Schalk, B. A. Schumm, A. Seiden, P. Spradlin, D. C. Williams, M. G. Wilson

University of California at Santa Cruz, Institute for Particle Physics, Santa Cruz, CA 95064, USA

J. Albert, E. Chen, G. P. Dubois-Felsmann, A. Dvoretskii, D. G. Hitlin, I. Narsky, T. Piatenko, F. C. Porter, A. Ryd, A. Samuel, S. Yang

California Institute of Technology, Pasadena, CA 91125, USA

S. Jayatilleke, G. Mancinelli, B. T. Meadows, M. D. Sokoloff

University of Cincinnati, Cincinnati, OH 45221, USA

T. Abe, F. Blanc, P. Bloom, S. Chen, W. T. Ford, U. Nauenberg, A. Olivas, P. Rankin, J. G. Smith, J. Zhang, L. Zhang University of Colorado, Boulder, CO 80309, USA

A. Chen, J. L. Harton, A. Soffer, W. H. Toki, R. J. Wilson, Q. Zeng Colorado State University, Fort Collins, CO 80523, USA

D. Altenburg, T. Brandt, J. Brose, M. Dickopp, E. Feltresi, A. Hauke, H. M. Lacker, R. Müller-Pfefferkorn, R. Nogowski, S. Otto, A. Petzold, J. Schubert, K. R. Schubert, R. Schwierz, B. Spaan, J. E. Sundermann

Technische Universität Dresden, Institut für Kern- und Teilchenphysik, D-01062 Dresden, Germany

D. Bernard, G. R. Bonneaud, F. Brochard, P. Grenier, S. Schrenk, Ch. Thiebaux, G. Vasileiadis, M. Verderi Ecole Polytechnique, LLR, F-91128 Palaiseau, France

D. J. Bard, P. J. Clark, D. Lavin, F. Muheim, S. Playfer, Y. Xie

University of Edinburgh, Edinburgh EH9 3JZ, United Kingdom

M. Andreotti, V. Azzolini, D. Bettoni, C. Bozzi, R. Calabrese, G. Cibinetto, E. Luppi, M. Negrini, L. Piemontese, A. Sarti

Università di Ferrara, Dipartimento di Fisica and INFN, I-44100 Ferrara, Italy

E. Treadwell

Florida A $6 M$ University, Tallahassee, FL 3230\%, USA

F. Anulli, R. Baldini-Ferroli, A. Calcaterra, R. de Sangro, G. Finocchiaro, P. Patteri, I. M. Peruzzi, M. Piccolo, A. Zallo

Laboratori Nazionali di Frascati dell'INFN, I-00044 Frascati, Italy

A. Buzzo, R. Capra, R. Contri, G. Crosetti, M. Lo Vetere, M. Macri, M. R. Monge, S. Passaggio, C. Patrignani, E. Robutti, A. Santroni, S. Tosi

Università di Genova, Dipartimento di Fisica and INFN, I-16146 Genova, Italy

S. Bailey, G. Brandenburg, K. S. Chaisanguanthum, M. Morii, E. Won

Harvard University, Cambridge, MA 02138, USA 
R. S. Dubitzky, U. Langenegger

Universität Heidelberg, Physikalisches Institut, Philosophenweg 12, D-69120 Heidelberg, Germany

W. Bhimji, D. A. Bowerman, P. D. Dauncey, U. Egede, J. R. Gaillard, G. W. Morton, J. A. Nash, M. B. Nikolich, G. P. Taylor Imperial College London, London, SWr 2AZ, United Kingdom

M. J. Charles, G. J. Grenier, U. Mallik

University of Iowa, Iowa City, IA 52242, USA

J. Cochran, H. B. Crawley, J. Lamsa, W. T. Meyer, S. Prell, E. I. Rosenberg, A. E. Rubin, J. Yi Iowa State University, Ames, IA 50011-3160, USA

M. Biasini, R. Covarelli, M. Pioppi

Università di Perugia, Dipartimento di Fisica and INFN, I-06100 Perugia, Italy

M. Davier, X. Giroux, G. Grosdidier, A. Höcker, S. Laplace, F. Le Diberder, V. Lepeltier, A. M. Lutz, T. C. Petersen, S. Plaszczynski, M. H. Schune, L. Tantot, G. Wormser

Laboratoire de l'Accélérateur Linéaire, F-91898 Orsay, France

C. H. Cheng, D. J. Lange, M. C. Simani, D. M. Wright

Lawrence Livermore National Laboratory, Livermore, CA 94550, USA

A. J. Bevan, C. A. Chavez, J. P. Coleman, I. J. Forster, J. R. Fry, E. Gabathuler, R. Gamet, D. E. Hutchcroft, R. J. Parry, D. J. Payne, R. J. Sloane, C. Touramanis

University of Liverpool, Liverpool L69 72E, United Kingdom

J. J. Back, ${ }^{1}$ C. M. Cormack, P. F. Harrison, ${ }^{1}$ F. Di Lodovico, G. B. Mohanty ${ }^{1}$

Queen Mary, University of London, E1 4NS, United Kingdom

C. L. Brown, G. Cowan, R. L. Flack, H. U. Flaecher, M. G. Green, P. S. Jackson, T. R. McMahon, S. Ricciardi, F. Salvatore, M. A. Winter

University of London, Royal Holloway and Bedford New College, Egham, Surrey TW20 0EX, United Kingdom

D. Brown, C. L. Davis

University of Louisville, Louisville, KY 40292, USA

J. Allison, N. R. Barlow, R. J. Barlow, P. A. Hart, M. C. Hodgkinson, G. D. Lafferty, A. J. Lyon, J. C. Williams

University of Manchester, Manchester M13 9PL, United Kingdom

A. Farbin, W. D. Hulsbergen, A. Jawahery, D. Kovalskyi, C. K. Lae, V. Lillard, D. A. Roberts

University of Maryland, College Park, MD 20742, USA

G. Blaylock, C. Dallapiccola, K. T. Flood, S. S. Hertzbach, R. Kofler, V. B. Koptchev, T. B. Moore, S. Saremi, H. Staengle, S. Willocq

University of Massachusetts, Amherst, MA 01003, USA

${ }^{1}$ Now at Department of Physics, University of Warwick, Coventry, United Kingdom 
R. Cowan, G. Sciolla, S. J. Sekula, F. Taylor, R. K. Yamamoto

Massachusetts Institute of Technology, Laboratory for Nuclear Science, Cambridge, MA 02139, USA

D. J. J. Mangeol, P. M. Patel, S. H. Robertson

McGill University, Montréal, QC, Canada H3A 2T8

A. Lazzaro, V. Lombardo, F. Palombo

Università di Milano, Dipartimento di Fisica and INFN, I-20133 Milano, Italy

J. M. Bauer, L. Cremaldi, V. Eschenburg, R. Godang, R. Kroeger, J. Reidy, D. A. Sanders, D. J. Summers, H. W. Zhao

University of Mississippi, University, MS 3867\%, USA

S. Brunet, D. Côté, P. Taras

Université de Montréal, Laboratoire René J. A. Lévesque, Montréal, QC, Canada H3C 3J7

H. Nicholson

Mount Holyoke College, South Hadley, MA 01075, USA

N. Cavallo, ${ }^{2}$ F. Fabozzi, ${ }^{2}$ C. Gatto, L. Lista, D. Monorchio, P. Paolucci, D. Piccolo, C. Sciacca

Università di Napoli Federico II, Dipartimento di Scienze Fisiche and INFN, I-80126, Napoli, Italy

M. Baak, H. Bulten, G. Raven, H. L. Snoek, L. Wilden

NIKHEF, National Institute for Nuclear Physics and High Energy Physics, NL-1009 DB Amsterdam, The Netherlands

C. P. Jessop, J. M. LoSecco

University of Notre Dame, Notre Dame, IN 46556, USA

T. Allmendinger, K. K. Gan, K. Honscheid, D. Hufnagel, H. Kagan, R. Kass, T. Pulliam, A. M. Rahimi, R. Ter-Antonyan, Q. K. Wong

Ohio State University, Columbus, OH 43210, USA

J. Brau, R. Frey, O. Igonkina, C. T. Potter, N. B. Sinev, D. Strom, E. Torrence

University of Oregon, Eugene, OR 97403, USA

F. Colecchia, A. Dorigo, F. Galeazzi, M. Margoni, M. Morandin, M. Posocco, M. Rotondo, F. Simonetto, R. Stroili, G. Tiozzo, C. Voci

Università di Padova, Dipartimento di Fisica and INFN, I-35131 Padova, Italy

M. Benayoun, H. Briand, J. Chauveau, P. David, Ch. de la Vaissière, L. Del Buono, O. Hamon, M. J. J. John, Ph. Leruste, J. Malcles, J. Ocariz, M. Pivk, L. Roos, S. T'Jampens, G. Therin

Universités Paris VI et VII, Laboratoire de Physique Nucléaire et de Hautes Energies, F-75252 Paris, France

P. F. Manfredi, V. Re

Università di Pavia, Dipartimento di Elettronica and INFN, I-27100 Pavia, Italy

\footnotetext{
${ }^{2}$ Also with Università della Basilicata, Potenza, Italy
} 


\author{
P. K. Behera, L. Gladney, Q. H. Guo, J. Panetta \\ University of Pennsylvania, Philadelphia, PA 19104, USA
}

C. Angelini, G. Batignani, S. Bettarini, M. Bondioli, F. Bucci, G. Calderini, M. Carpinelli, F. Forti, M. A. Giorgi, A. Lusiani, G. Marchiori, F. Martinez-Vidal, ${ }^{3}$ M. Morganti, N. Neri, E. Paoloni, M. Rama, G. Rizzo, F. Sandrelli, J. Walsh

Università di Pisa, Dipartimento di Fisica, Scuola Normale Superiore and INFN, I-56127 Pisa, Italy

M. Haire, D. Judd, K. Paick, D. E. Wagoner

Prairie View A\&M University, Prairie View, TX 77446, USA

N. Danielson, P. Elmer, Y. P. Lau, C. Lu, V. Miftakov, J. Olsen, A. J. S. Smith, A. V. Telnov

Princeton University, Princeton, NJ 08544, USA

F. Bellini, G. Cavoto, ${ }^{4}$ R. Faccini, F. Ferrarotto, F. Ferroni, M. Gaspero, L. Li Gioi, M. A. Mazzoni, S. Morganti, M. Pierini, G. Piredda, F. Safai Tehrani, C. Voena Università di Roma La Sapienza, Dipartimento di Fisica and INFN, I-00185 Roma, Italy

S. Christ, G. Wagner, R. Waldi

Universität Rostock, D-18051 Rostock, Germany

T. Adye, N. De Groot, B. Franek, N. I. Geddes, G. P. Gopal, E. O. Olaiya

Rutherford Appleton Laboratory, Chilton, Didcot, Oxon, OX11 0QX, United Kingdom

R. Aleksan, S. Emery, A. Gaidot, S. F. Ganzhur, P.-F. Giraud, G. Hamel de Monchenault, W. Kozanecki, M. Legendre, G. W. London, B. Mayer, G. Schott, G. Vasseur, Ch. Yèche, M. Zito

DSM/Dapnia, CEA/Saclay, F-91191 Gif-sur-Yvette, France

M. V. Purohit, A. W. Weidemann, J. R. Wilson, F. X. Yumiceva

University of South Carolina, Columbia, SC 29208, USA

D. Aston, R. Bartoldus, N. Berger, A. M. Boyarski, O. L. Buchmueller, R. Claus, M. R. Convery, M. Cristinziani, G. De Nardo, D. Dong, J. Dorfan, D. Dujmic, W. Dunwoodie, E. E. Elsen, S. Fan,

R. C. Field, T. Glanzman, S. J. Gowdy, T. Hadig, V. Halyo, C. Hast, T. Hryn'ova, W. R. Innes,

M. H. Kelsey, P. Kim, M. L. Kocian, D. W. G. S. Leith, J. Libby, S. Luitz, V. Luth, H. L. Lynch,

H. Marsiske, R. Messner, D. R. Muller, C. P. O'Grady, V. E. Ozcan, A. Perazzo, M. Perl, S. Petrak,

B. N. Ratcliff, A. Roodman, A. A. Salnikov, R. H. Schindler, J. Schwiening, G. Simi, A. Snyder, A. Soha, J. Stelzer, D. Su, M. K. Sullivan, J. Va'vra, S. R. Wagner, M. Weaver, A. J. R. Weinstein,

W. J. Wisniewski, M. Wittgen, D. H. Wright, A. K. Yarritu, C. C. Young

Stanford Linear Accelerator Center, Stanford, CA 94309, USA

P. R. Burchat, A. J. Edwards, T. I. Meyer, B. A. Petersen, C. Roat

Stanford University, Stanford, CA 94305-4060, USA

S. Ahmed, M. S. Alam, J. A. Ernst, M. A. Saeed, M. Saleem, F. R. Wappler

State University of New York, Albany, NY 12222, USA

\footnotetext{
${ }^{3}$ Also with IFIC, Instituto de Física Corpuscular, CSIC-Universidad de Valencia, Valencia, Spain

${ }^{4}$ Also with Princeton University, Princeton, USA
} 
W. Bugg, M. Krishnamurthy, S. M. Spanier

University of Tennessee, Knoxville, TN 37996, USA

R. Eckmann, H. Kim, J. L. Ritchie, A. Satpathy, R. F. Schwitters

University of Texas at Austin, Austin, TX 78712, USA

J. M. Izen, I. Kitayama, X. C. Lou, S. Ye

University of Texas at Dallas, Richardson, TX 75083, USA

F. Bianchi, M. Bona, F. Gallo, D. Gamba

Università di Torino, Dipartimento di Fisica Sperimentale and INFN, I-10125 Torino, Italy

L. Bosisio, C. Cartaro, F. Cossutti, G. Della Ricca, S. Dittongo, S. Grancagnolo, L. Lanceri, P. Poropat, ${ }^{5}$ L. Vitale, G. Vuagnin

Università di Trieste, Dipartimento di Fisica and INFN, I-34127 Trieste, Italy

R. S. Panvini

Vanderbilt University, Nashville, TN 37235, USA

Sw. Banerjee, C. M. Brown, D. Fortin, P. D. Jackson, R. Kowalewski, J. M. Roney, R. J. Sobie University of Victoria, Victoria, BC, Canada V8W $3 P 6$

H. R. Band, B. Cheng, S. Dasu, M. Datta, A. M. Eichenbaum, M. Graham, J. J. Hollar, J. R. Johnson, P. E. Kutter, H. Li, R. Liu, A. Mihalyi, A. K. Mohapatra, Y. Pan, R. Prepost, P. Tan, J. H. von Wimmersperg-Toeller, J. Wu, S. L. Wu, Z. Yu

University of Wisconsin, Madison, WI 53706, USA

M. G. Greene, H. Neal

Yale University, New Haven, CT 06511, USA

\footnotetext{
${ }^{5}$ Deceased
} 
The hadronic decay ${ }^{6} D_{s}^{*+} \rightarrow D_{s}^{+} \pi^{0}$ violates isospin conservation and should be suppressed with respect to the radiative decay $D_{s}^{*+} \rightarrow D_{s}^{+} \gamma$. Cho and Wise [1] have calculated the decay rate for $D_{s}^{*+} \rightarrow D_{s}^{+} \pi^{0}$ using chiral perturbation theory; they describe the process as an isospin conserving decay $D_{s}^{*+} \rightarrow D_{s}^{+} \eta$ followed by the conversion of the virtual $\eta$ meson into a $\pi^{0}$ meson through mixing. The radiative process $D_{s}^{*+} \rightarrow D_{s}^{+} \gamma$ proceeds via magnetic transitions to which both the coupling to the charm quark and the light quark contribute. Since a neutral pion or kaon do not couple to the photon, only virtual states with a charged pion or kaon contribute to one-loop corrections of the magnetic moments. The difference in the kaon and pion masses naturally leads to $\mathrm{SU}(3)$ flavor-symmetry breaking.

A previous measurement of the ratio $\Gamma\left(D_{s}^{*+} \rightarrow D_{s}^{+} \pi^{0}\right) / \Gamma\left(D_{s}^{*+} \rightarrow D_{s}^{+} \gamma\right)$ by the CLEO Collaboration obtained a result of $0.062_{-0.018}^{+0.020}$ (stat) \pm 0.022 (syst) using $14.7_{-4.0}^{+4.6} D_{s}^{*+} \rightarrow D_{s}^{+} \pi^{0}$ events [2].

The analysis we present here, using $90.4 \mathrm{fb}^{-1}$ of data recorded by the BABAR detector at the PEP-II asymmetric $e^{+} e^{-}$storage ring, represents a significant improvement in precision and is particularly relevant given the recent observations of two new $D_{s}^{+}$meson states $[3,4]$. The data have been recorded at and approximately $40 \mathrm{MeV}$ below the $\Upsilon(4 S)$ resonance.

The BABAR detector is described in detail elsewhere [5]. Charged particles are detected and their momenta measured by a silicon vertex tracker (SVT) consisting of five layers of double-sided silicon strip sensors and a cylindrical 40-layer drift chamber $(\mathrm{DCH})$, both operating within a $1.5 \mathrm{~T}$ solenoidal magnetic field. Charged particle identification is provided by energy loss measurements in the SVT and DCH and by light recorded in an internally reflecting ring imaging Cherenkov detector (DIRC). Neutral particles are identified and their energies measured by an electromagnetic calorimeter (EMC) composed of $6580 \mathrm{CsI}(\mathrm{Tl})$ crystals.

$D_{s}^{+}$mesons are reconstructed using the decay $D_{s}^{+} \rightarrow \phi \pi^{+}, \phi \rightarrow K^{+} K^{-}$. Kaons are identified by combining the energy deposited in the SVT and DCH with the DIRC information. Tracks not identified as kaons according to the PID criteria are considered as pions.

Our selection criteria are chosen such that the signal significance $S^{2} /(S+B)$ of the channel $D_{s}^{*+} \rightarrow D_{s}^{+} \pi^{0}$ is maximized. $S$ and $B$ refer to the expected numbers of signal and background events, respectively, which are obtained from a sample of Monte Carlo events.

All combinations of $K^{+} K^{-} \pi^{+}$candidates have been required to successfully fit to a common vertex; only combinations with a $K^{+} K^{-}$mass within $8 \mathrm{MeV} / c^{2}$ from the nominal $\phi$ mass [6] have been retained.

The scaled momentum is defined as $x_{p}\left(D_{s}^{+}\right)=p^{*}\left(D_{s}^{+}\right) / p_{\max }^{*}\left(D_{s}^{+}\right)$, where $p^{*}\left(D_{s}^{+}\right)$is the momentum of the $D_{s}^{+}$candidates in the center-of-mass frame, and $p_{\max }^{*}\left(D_{s}^{+}\right)=\sqrt{E_{\text {beam }}^{*}-m\left(D_{s}^{+}\right)^{2}}$ is its maximum value. Combinations of $\phi$ and $\pi^{+}$candidates are retained if the scaled momentum is 0.6 or greater. Since the kinematics of the $c \bar{c}$ fragmentation process produce charmed mesons with high momenta, this reduces the combinatorial background.

We exploit the longitudinal polarization of the $\phi$ meson to reduce continuum background by requiring that the absolute value of the cosine of the helicity angle $\theta_{H}$, which is defined as the angle between the $\phi$ momentum direction in the $D_{s}^{+}$rest frame and the momentum direction of one of the kaons in the $\phi$ rest frame, is 0.3 or greater.

The $K^{+} K^{-} \pi^{+}$invariant mass distribution is shown in Fig. 1. By fitting the sum of a double Gaussian function (to represent the signal) and a third-order polynomial (to represent the background) to this distribution, we find $73500 \pm 300$ events. $D_{s}^{+}$candidates are retained if their invariant mass is within $12 \mathrm{MeV} / c^{2}$ from the nominal $D_{s}^{+}$mass [6].

\footnotetext{
${ }^{6}$ Inclusion of the charge conjugated state is assumed throughout this paper.
} 


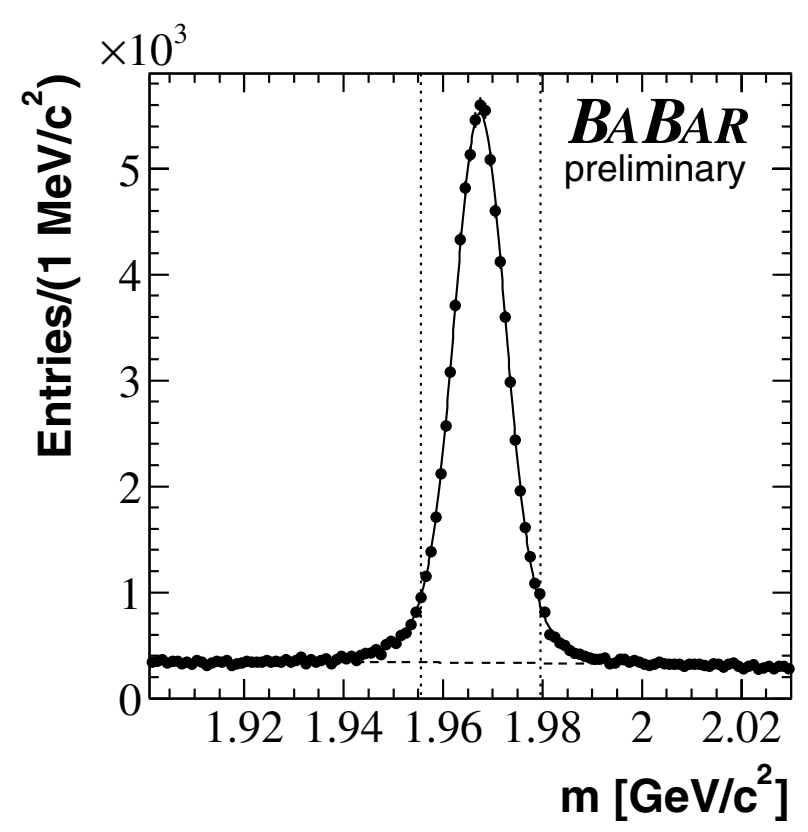

Figure 1: $K^{+} K^{-} \pi^{+}$mass distribution. The dots represent data points. The solid line shows the fitted function. The dashed line indicates the portion of the fit associated with the background. $D_{s}^{+}$candidates are selected from the region between the vertical dotted lines.

Each $\pi^{0}$ candidate is reconstructed by combining two photon candidates. Each photon candidate consists of a calorimeter cluster which is not associated with a charged track and has an energy in the laboratory frame of at least $45 \mathrm{MeV}$. Additionally, to help remove the background from hadrons, we require the lateral moment [7], which describes the shape of the electromagnetic shower in the calorimeter, to be less than 0.55 .

The $\pi^{0}$ candidates are retained if they have a momentum $p^{*}$ in the center-of-mass frame greater than $150 \mathrm{MeV} / c$. Furthermore, the absolute value of the cosine of the decay angle $\theta^{*}\left(\pi^{0}\right)$, which is defined as the angle between the direction of one of the photons in the $\pi^{0}$ rest frame and the direction of the $\pi^{0}$ candidate in the center-of-mass frame, is required to be less than 0.85. For the isotropic $\pi^{0}$ decay, the $\left|\cos \theta^{*}\left(\pi^{0}\right)\right|$ distribution is flat, while it peaks near 1 for random $\gamma \gamma$ combinations.

Only $\gamma \gamma$ pairs inside a mass window are retained. This window is defined as the region where a function fitted to the $\gamma \gamma$ mass distribution of true $\pi^{0} \rightarrow \gamma \gamma$ Monte Carlo events exceeds 0.2 its maximum value, which accommodates the asymmetric shape of the $\gamma \gamma$ mass distribution and takes different detector calibrations into account. A kinematic fit is applied to the surviving $\gamma \gamma$ pairs, constrained to the nominal $\pi^{0}$ mass.

After combining the $D_{s}^{+}$and $\pi^{0}$ candidates to reconstruct the decay $D_{s}^{*+} \rightarrow D_{s}^{+} \pi^{0}$, we have fitted a function to the mass difference $\Delta m\left(D_{s}^{+} \pi^{0}\right)=m\left(K^{+} K^{-} \pi^{+} \pi^{0}\right)-m\left(K^{+} K^{-} \pi^{+}\right)$. The function is the sum of a double Gaussian function to represent the signal and

$$
f_{1}(\Delta m)=N \cdot\left(1-\exp \left(-\frac{\Delta m-m\left(\pi^{0}\right)}{\mu}\right)\right) \cdot\left(\Delta m^{2}+a \Delta m+b\right)
$$

(where $m\left(\pi^{0}\right)$ is the $\pi^{0}$ mass, while $N, \mu, a$, and $b$ are free fit parameters) to represent the 

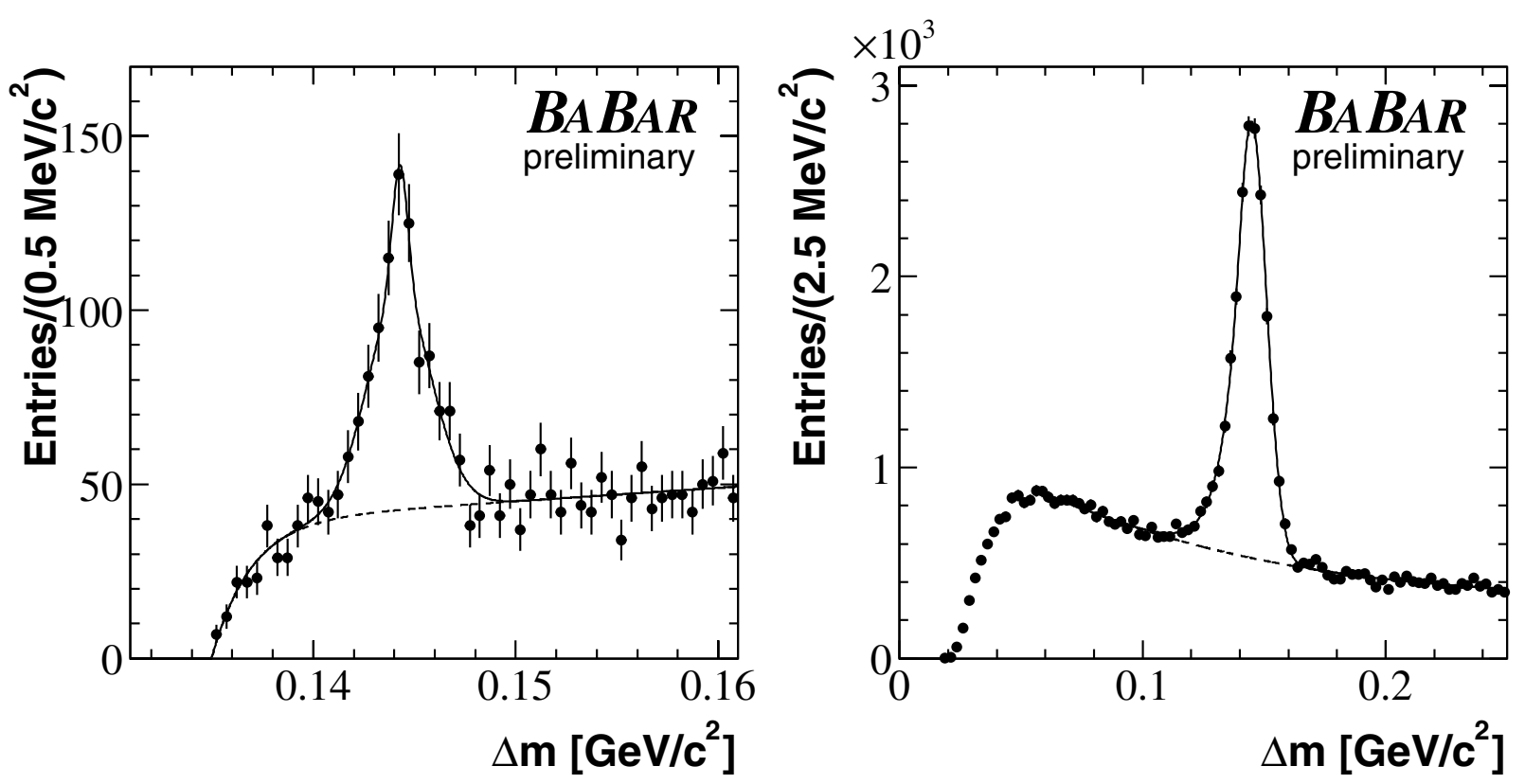

Figure 2: $D_{s}^{*+}$ signals: (a) $m\left(K^{+} K^{-} \pi^{+} \pi^{0}\right)-m\left(K^{+} K^{-} \pi^{+}\right)$; (b) $m\left(K^{+} K^{-} \pi^{+} \gamma\right)-m\left(K^{+} K^{-} \pi^{+}\right)$. The dots represent data points. The solid line shows the fitted function. The dashed line indicates the portion of the fit associated with the background.

background. The exponential term in the background function models the kinematic threshold; it is close to 1 in the signal region.

The result of this fit is shown in Fig. 2(a). We find a signal event yield of $560 \pm 40$.

For the reconstruction of the decay $D_{s}^{*+} \rightarrow D_{s}^{+} \gamma$, calorimeter clusters that are not associated with a charged track are considered photon candidates if they fulfill the following requirements. The energy must be $50 \mathrm{MeV}$ or greater in the laboratory frame and $100 \mathrm{MeV}$ or greater in the center-of-mass frame. The lateral moment must be less than 0.8. To reduce the background of photons coming from $\pi^{0}$ decays, a photon candidate is discarded if it forms a $\pi^{0}$ candidate with any other photon candidate in the same event. Here, a $\gamma \gamma$ combination is considered a $\pi^{0}$ candidate if the invariant mass is in the range $115<m(\gamma \gamma)<155 \mathrm{MeV} / c^{2}$ and has an energy of at least $200 \mathrm{MeV}$ in the center-of-mass frame.

To obtain the $D_{s}^{*+} \rightarrow D_{s}^{+} \gamma$ signal event yield, we have fitted a function to the mass difference $\Delta m\left(D_{s}^{+} \gamma\right)=m\left(K^{+} K^{-} \pi^{+} \gamma\right)-m\left(K^{+} K^{-} \pi^{+}\right)$. This function is a sum of a "Crystal Ball" function [8]

$$
f_{2}(\Delta m)=N \cdot \begin{cases}A\left(B-\frac{\Delta m-\mu}{\sigma}\right)^{-n} & \text { if }(\Delta m-\mu) / \sigma \leq \alpha \\ \exp \left(-\frac{(\Delta m-\mu)^{2}}{2 \sigma^{2}}\right) & \text { if }(\Delta m-\mu) / \sigma \geq \alpha\end{cases}
$$

(where $N, \mu, \sigma, n$, and $\alpha$ are free fit parameters, and $A$ and $B$ are chosen such that the function and its first derivative are continuous at $(\Delta m-\mu) / \sigma=\alpha)$ for the signal, and a third-order polynomial for the background. The fit result is shown in Fig. 2(b). We find $15600 \pm 200$ signal events.

To determine the reconstruction efficiencies, two samples of signal Monte Carlo events for the decays $D_{s}^{*+} \rightarrow D_{s}^{+} \pi^{0}$ and $D_{s}^{*+} \rightarrow D_{s}^{+} \gamma$, each of which consists of 30000 events, have been used. The Monte Carlo events have been analyzed using the same procedure as for real data. By calculating the ratio of reconstructed to generated event numbers, we find efficiencies of $\epsilon\left(D_{s}^{+} \pi^{0}\right)=$ 

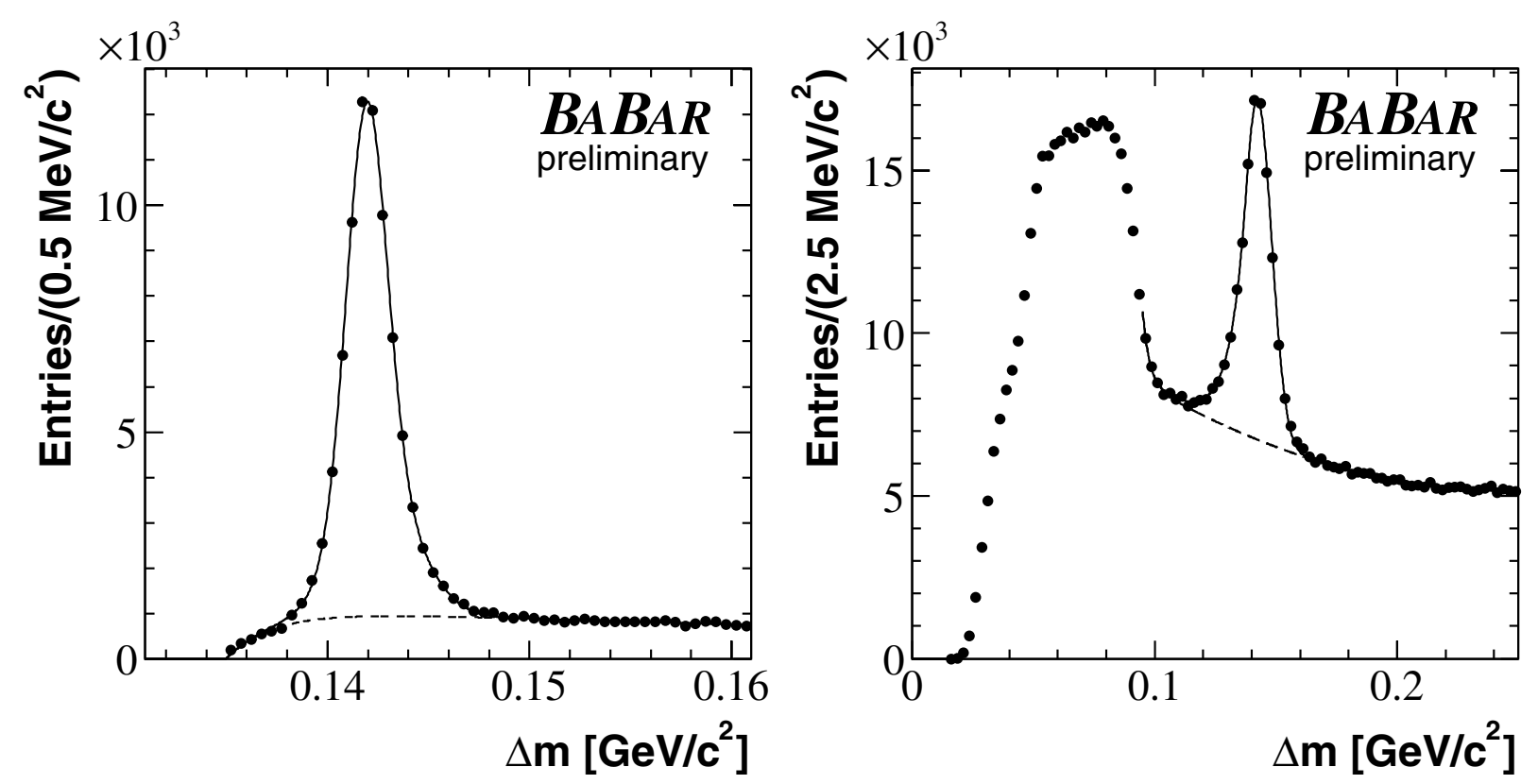

Figure 3: $D^{* 0}$ signals: (a) $m\left(K^{-} \pi^{+} \pi^{0}\right)-m\left(K^{-} \pi^{+}\right)$; (b) $m\left(K^{-} \pi^{+} \gamma\right)-m\left(K^{-} \pi^{+}\right)$. The dots represent data points. The solid line shows the fitted function. The dashed line indicates the portion of the fit associated with the background.

$0.041 \pm 0.002$ and $\epsilon\left(D_{s}^{+} \gamma\right)=0.071 \pm 0.002$ for the two $D_{s}^{*+}$ decay modes. The efficiency ratio is $\epsilon\left(D_{s}^{+} \pi^{0}\right) / \epsilon\left(D_{s}^{+} \gamma\right)=0.58 \pm 0.03$.

For completeness, and as a check of our efficiency calculations, we have also measured the ratio $\Gamma\left(D^{* 0} \rightarrow D^{0} \pi^{0}\right) / \Gamma\left(D^{* 0} \rightarrow D^{0} \gamma\right)$ using the same selection criteria for the $\pi^{0}$ and photon candidates as in the reconstruction of $D_{s}^{*+} \rightarrow D_{s}^{+} \pi^{0}$ and $D_{s}^{*+} \rightarrow D_{s}^{+} \gamma$. To reconstruct the decay $D^{0} \rightarrow K^{-} \pi^{+}$, combinations of $K^{-}$and $\pi^{+}$candidates are required to successfully fit to a common vertex, and the scaled momentum of the resulting $D^{0}$ candidate must be 0.6 or greater. Fitting the sum of a double Gaussian function and a third-order polynomial to the resulting $K^{-} \pi^{+}$invariant mass distribution, we find $(996.0 \pm 1.5) \times 10^{3}$ signal events. $K^{-} \pi^{+}$combinations are retained if their mass differs by less than $17 \mathrm{MeV} / c^{2}$ from the nominal $D^{0}$ mass [6].

The $D^{0}$ candidates are combined with all $\pi^{0}$ candidates; the resulting mass difference $\Delta m\left(D^{0} \pi^{0}\right)=$ $m\left(K^{-} \pi^{+} \pi^{0}\right)-m\left(K^{-} \pi^{+}\right)$is shown in Fig. 3(a). A fit using a double Gaussian for the signal and the function shown in Eq. 1 for the background yields $69000 \pm 400$ signal events.

The $D^{0}$ candidates are then combined with all photon candidates producing the distribution of the mass difference $\Delta m\left(D^{0} \gamma\right)=m\left(K^{-} \pi^{+} \gamma\right)-m\left(K^{-} \pi^{+}\right)$shown in Fig. 3(b). In this case the signal peak of $D^{* 0} \rightarrow D^{0} \gamma$ is close to a large bump due to the reflection of $D^{* 0} \rightarrow D^{0} \pi^{0}$ where one photon comes from a $\pi^{0}$ decay. We therefore model the background using the function

$$
f_{3}(\Delta m)=N \cdot\left(1+\exp \left(-\frac{\Delta m-m\left(\pi^{0}\right)}{\mu}\right)\right) \cdot\left(\Delta m^{2}+a \Delta m+b\right) .
$$

(Note that this function is similar to Eq. 1, but differs in the sign of the exponential term.)

The signal is modeled by a "Crystal Ball" function (Eq. 2). The resulting fitted signal consists of $67900 \pm 700$ events. 
Table 1: Summary of systematic uncertainties on the branching ratio $\Gamma\left(D_{s}^{*+} \rightarrow D_{s}^{+} \pi^{0}\right) / \Gamma\left(D_{s}^{*+} \rightarrow D_{s}^{+} \gamma\right)$.

\begin{tabular}{lr}
\hline Signal Monte Carlo Statistics & $5.0 \%$ \\
Monte Carlo Simulation Uncertainty & $3.6 \%$ \\
$D_{s}^{+}$Background & $3.2 \%$ \\
$\pi^{0}$ Background & $3.1 \%$ \\
Momentum Dependence & $6.8 \%$ \\
\hline Total & $10.2 \%$ \\
\hline
\end{tabular}

Table 2: Summary of systematic uncertainties on the branching ratio $\Gamma\left(D^{* 0} \rightarrow D^{0} \pi^{0}\right) / \Gamma\left(D^{* 0} \rightarrow D^{0} \gamma\right)$.

\begin{tabular}{ll}
\hline Signal Monte Carlo Statistics & $5.4 \%$ \\
Monte Carlo Simulation Uncertainty & $3.8 \%$ \\
$D^{0}$ Background & $0.1 \%$ \\
Momentum Dependence & $2.8 \%$ \\
\hline Total & $7.2 \%$ \\
\hline
\end{tabular}

We determine the efficiency ratio by the reconstruction of 30000 signal Monte Carlo events for each of the channels $D^{* 0} \rightarrow D^{0} \pi^{0}$ and $D^{* 0} \rightarrow D^{0} \gamma$. Efficiencies of $\epsilon\left(D^{0} \pi^{0}\right)=0.037 \pm 0.002$ and $\epsilon\left(D^{0} \gamma\right)=0.064 \pm 0.002$, and an efficiency ratio of $\epsilon\left(D^{0} \pi^{0}\right) / \epsilon\left(D^{0} \gamma\right)=0.58 \pm 0.03$ are found. This is in perfect agreement with the value of $\epsilon\left(D_{s}^{+} \pi^{0}\right) / \epsilon\left(D_{s}^{+} \gamma\right)=0.58 \pm 0.03$. A ratio of $\Gamma\left(D^{* 0} \rightarrow\right.$ $\left.D^{0} \pi^{0}\right) / \Gamma\left(D^{* 0} \rightarrow D^{0} \gamma\right)=1.740 \pm 0.020$ (statistical error only) is obtained, which agrees with the world average of $\Gamma\left(D^{* 0} \rightarrow D^{0} \pi^{0}\right) / \Gamma\left(D^{* 0} \rightarrow D^{0} \gamma\right)=1.625 \pm 0.200[6]$.

Various sources of systematic uncertainties have been studied; they are summarized in Tables 1 and 2 .

To verify that the Monte Carlo events model the data correctly, we have studied $\tau$ decays with one or two $\pi^{0}$ mesons in the final state to obtain energy-dependent Monte Carlo efficiency correction functions for $\pi^{0}$ mesons and photons. While no correction is necessary, the errors on the correction functions represent uncertainties of the Monte Carlo model and are used as systematic uncertainties.

To test for uncertainties in the background shape of the mass difference distributions, we have considered left and right sidebands in the $D_{s}^{+}, D^{0}$, and $\pi^{0}$ masses. The widths of the sidebands have been chosen such that the number of events in each sideband corresponds to the expected number of background events in the signal region. We have fitted the same functions used to determine the signal yields to the mass difference distributions of the sideband samples. Any discrepancy in the yield between data and Monte Carlo simulation is considered a systematic uncertainty.

We have repeated the measurements of $\Gamma\left(D_{s}^{*+} \rightarrow D_{s}^{+} \pi^{0}\right) / \Gamma\left(D_{s}^{*+} \rightarrow D_{s}^{+} \gamma\right)$ and $\Gamma\left(D^{* 0} \rightarrow\right.$ $\left.D^{0} \pi^{0}\right) / \Gamma\left(D^{* 0} \rightarrow D^{0} \gamma\right)$ for candidates restricted to bins of $p^{*}$ (encompassing the range $3<p^{*}<$ $5 \mathrm{GeV} / c$ ) by repeating the mass difference fit to the subsample of candidates within each bin. By fitting both a constant function and a first-order polynomial to each branching ratio as a function of $p^{*}$, we have verified that the measured branching ratios are independent of $p^{*}$. Nevertheless, 
Table 3: Summary of preliminary results. The first errors are statistical, the second represent systematic uncertainties.

\begin{tabular}{ll}
\hline$\Gamma\left(D_{s}^{*+} \rightarrow D_{s}^{+} \pi^{0}\right) / \Gamma\left(D_{s}^{*+} \rightarrow D_{s}^{+} \gamma\right)$ & $0.0621 \pm 0.0049 \pm 0.0063$ \\
$\mathcal{B}\left(D_{s}^{*+} \rightarrow D_{s}^{+} \pi^{0}\right)$ & $0.0585 \pm 0.0043 \pm 0.0056$ \\
$\mathcal{B}\left(D_{s}^{*+} \rightarrow D_{s}^{+} \gamma\right)$ & $0.9415 \pm 0.0043 \pm 0.0056$ \\
\hline$\Gamma\left(D^{* 0} \rightarrow D^{0} \pi^{0}\right) / \Gamma\left(D^{* 0} \rightarrow D^{0} \gamma\right)$ & $1.740 \pm 0.020 \pm 0.125$ \\
$\mathcal{B}\left(D^{* 0} \rightarrow D^{0} \pi^{0}\right)$ & $0.6351 \pm 0.0027 \pm 0.0166$ \\
$\mathcal{B}\left(D^{* 0} \rightarrow D^{0} \gamma\right)$ & $0.3649 \pm 0.0027 \pm 0.0166$ \\
\hline
\end{tabular}

we assume conservatively that the first-order polynomials arise from unknown momentum dependencies of the efficiencies which do not cancel in the branching ratios. Taking the momentum distributions of the $D_{s}^{*+}$ and $D^{* 0}$ mesons into account, we use the difference between the branching ratio represented by the constant function and the first-order polynomial for each distribution as a systematic uncertainty.

The branching ratios are shown in Table 3. By assuming that the $D_{s}^{*+}$ meson decays only to $D_{s}^{+} \pi^{0}$ and $D_{s}^{+} \gamma$, i.e. $\mathcal{B}\left(D_{s}^{*+} \rightarrow D_{s}^{+} \pi^{0}\right)+\mathcal{B}\left(D_{s}^{*+} \rightarrow D_{s}^{+} \gamma\right)=1$, and that the $D^{* 0}$ meson decays only to $D^{0} \pi^{0}$ and $D^{0} \gamma$, i.e. $\mathcal{B}\left(D^{* 0} \rightarrow D^{0} \pi^{0}\right)+\mathcal{B}\left(D^{* 0} \rightarrow D^{0} \gamma\right)=1$, it is possible to calculate the branching fractions, which are also listed in Table 3 .

In summary, we have measured a preliminary value for the branching ratio $\Gamma\left(D_{s}^{*+} \rightarrow D_{s}^{+} \pi^{0}\right) /$ $\Gamma\left(D_{s}^{*+} \rightarrow D_{s}^{+} \gamma\right)=0.0621 \pm 0.0049$ (stat) \pm 0.0063 (syst), which is consistent with the previous measurement [2], but has higher precision. We have also measured $\Gamma\left(D^{* 0} \rightarrow D^{0} \pi^{0}\right) / \Gamma\left(D^{* 0} \rightarrow\right.$ $\left.D^{0} \gamma\right)=1.740 \pm 0.020$ (stat) \pm 0.125 (syst), which is more precise than, and in agreement with, the world average [6].

The branching fraction $\mathcal{B}\left(D_{s}^{*+} \rightarrow D_{s}^{+} \pi^{0}\right)=0.0585 \pm 0.0043$ (stat) \pm 0.0056 (syst) is larger than the theoretical prediction, which is in the $1-3 \%$ range [1]. However, it should be noted that the theory predicts a strong correlation between $\mathcal{B}\left(D_{s}^{*+} \rightarrow D_{s}^{+} \pi^{0}\right)$ and $\mathcal{B}\left(D^{*+} \rightarrow D^{+} \gamma\right)$; for $\mathcal{B}\left(D^{*+} \rightarrow\right.$ $\left.D^{+} \gamma\right) \lesssim 1 \%, \mathcal{B}\left(D_{s}^{*+} \rightarrow D_{s}^{+} \pi^{0}\right)$ is expected to be greatly enhanced.

We are grateful for the excellent luminosity and machine conditions provided by our PEP-II colleagues, and for the substantial dedicated effort from the computing organizations that support BABAR. The collaborating institutions wish to thank SLAC for its support and kind hospitality. This work is supported by DOE and NSF (USA), NSERC (Canada), IHEP (China), CEA and CNRS-IN2P3 (France), BMBF and DFG (Germany), INFN (Italy), FOM (The Netherlands), NFR (Norway), MIST (Russia), and PPARC (United Kingdom). Individuals have received support from CONACyT (Mexico), A. P. Sloan Foundation, Research Corporation, and Alexander von Humboldt Foundation.

\section{References}

[1] P. L. Cho and M. B. Wise, Phys. Rev. D 49, 6228 (1994).

[2] CLEO Collaboration, J. Gronberg et al., Phys. Rev. Lett. 75, 3232 (1995).

[3] BABAR Collaboration, B. Aubert et al., Phys. Rev. Lett. 90, 242001 (2003). 
[4] CLEO Collaboration, D. Besson et al., Phys. Rev. D 68, 032002 (2003).

[5] BABAR Collaboration, B. Aubert et al., Nucl. Instr. Meth. A 479, 1 (2002).

[6] Particle Data Group, S. Eidelman et al., Phys. Lett. B 592, 1 (2004).

[7] A. Drescher et al., Nucl. Instr. Meth. A 237, 464 (1985).

[8] Crystal Ball Collaboration, D. Antreasyan et al., Crystal Ball Note 321 (1983). 